Research Paper

\title{
Efficacy of Second-line Tyrosine Kinase Inhibitors in the Treatment of Metastatic Advanced Non-small-cell Lung Cancer Harboring Exon 19 and 21 EGFR Mutations
}

\author{
Zhen Zheng ${ }^{1 *}$, Xiance Jin ${ }^{1 *}$, Baochai Lin ${ }^{1}$, Huafang Su${ }^{1}$, Hanbin Chen ${ }^{1}$, Shaoran Fei ${ }^{1}$, Lihao Zhao ${ }^{1}$, Xia \\ Deng ${ }^{1}$, Deyao $\mathrm{Xie}^{2 \bowtie}$, Congying Xie $\mathrm{X}^{1 凶}$ \\ 1. Department of Radiotherapy and Chemotherapy, the First Affiliated Hospital of Wenzhou Medical University, Wenzhou, China, 325000; \\ 2. Department of Thoracic Surgery, the First Affiliated Hospital of Wenzhou Medical University, Wenzhou, China, 325000. \\ * These authors contributed equally. \\ $\triangle$ Corresponding authors: Dr. Deyao Xie, MD, Department of Thoracic Surgery, the First Affiliated Hospital of Wenzhou Medical University, Wenzhou, China, \\ 325000. Phone: 0086-13506667375, Fax: 0086-577-55578999-667375, E-mail: 2590637486@qq.com. Dr. Congying Xie, PhD, Department of Radiotherapy and \\ Chemotherapy, The First Affiliated Hospital of Wenzhou Medical University, No.2 Fuxue Lane, Wenzhou, China, 325000. Phone: (0086)13867711881, Fax: \\ 0086-577-55578999-611881, E-mail: wzxiecongying@163.com.
}

(c) Ivyspring International Publisher. This is an open access article distributed under the terms of the Creative Commons Attribution (CC BY-NC) license (https://creativecommons.org/licenses/by-nc/4.0/). See http://ivyspring.com/terms for full terms and conditions.

Received: 2016.07.24; Accepted: 2016.10.29; Published: 2017.02.15

\begin{abstract}
Background: Although superior clinical benefits of first-line epidermal growth factor receptor (EGFR) tyrosine kinase inhibitors (TKIs) in the treatment of advanced non-small-cell lung cancer (NSCLC) had been reported with different sensitivity, the sensitivity of second-line TKIs in NSCLC patients with different EFGR mutations was unknown. The purpose of this study is to investigate the clinical outcome of second-line EGFR-TKIs in the treatment of NSCLC patients according to different EGFR genotypes.

Methods: The treatment outcomes of 166 NSCLC patients with different EGFR mutations treated by second-line TKIs were retrospectively reviewed. The efficacy was evaluated with Pearson chi-square or Fisher's exact tests, Log-rank test and Cox proportional hazards model.

Results: The disease control rate (DCR) and objective response rate (ORR) of enrolled NSCLC patients were $77.7 \%$ and $11.4 \%$, respectively. The exon 19 deletion group had a significantly longer median progression-free survival (PFS) (6.7 vs. 4.5 months, $P=0.002)$ and overall survival $(\mathrm{OS})$ (13.7 vs. 11.7 months, $\mathrm{P}=0.02$ ) compared with the exon 19 L858R mutation group for NSCLC patients, as well for patients with brain metastasis [PFS: (6.7 vs. 3.9 months, $p<0.001)$, OS: (13.7 vs. 7.9 months, $p=0.006)]$. No significant difference on PFS and OS was observed between exon 19 deletion and L858R mutation group for patients with bone metastasis. EGFR genotype and ECOG PS were independent predictors of PFS. Never smoking, exon 19 deletion, EGOC PS (0-1) and no brain metastasis were correlated with longer OS. No significant difference on side effect between exon 19 and 21 mutation group was observed.

Conclusions: NSCLC patients harboring exon 19 deletion achieved better PFS and OS than those with L858R mutation, indicating that EGFR mutation is a significant prognostic factor for advanced NSCLC patients with and without brain metastasis receiving second-line EGFR-TKIs treatment.
\end{abstract}

Key words: Non-small-cell lung cancer; Epidermal growth factor receptor; Tyrosine kinase inhibitors; Brain metastasis; Bone metastasis

\section{Introduction}

Lung cancer is the leading cause of cancer related deaths worldwide and in China. ${ }^{1,2}$ Non-small-cell lung cancer (NSCLC) accounts for approximately $87 \%$ of all lung cancer cases. ${ }^{3,4}$ The overall prognosis of NSCLC remains poor due to approximately $25-30 \%$ of patients present with locally advanced disease upon their initial diagnosis, whereas $40-50 \%$ patients present with metastatic 
disease $^{5}$. The appearance of small-molecule tyrosine kinase inhibitors (TKIs) of epidermal growth factor receptor (EGFR), such as gefitinib and erlotinib, have shown antitumor activity in NSCLC patients, especially in those with EGFR mutations. EGFR mutational status has become the most important determining factor of clinical response to TKIs. Results from phase II and phase III randomized-controlled trials (RCTs) demonstrated superior clinical benefits of TKIs compared with chemotherapy as a first-line therapy for metastatic NSCLC patients. ${ }^{6}$

It was found that sensitivity to EGFR TKI is associated with somatic mutations in the EGFR genes. The EGFR mutations were discovered in the first four exons, i.e. exons 18-21 of the tyrosine kinase domain of EGFR. ${ }^{7-9}$ Two major activating mutations in EGFR, which account for almost $85 \%$ of all clinically important mutations related to EGFR TKI sensitivity, are an in-frame deletion of exon 19 encompassing the amino acids from codons L747 to E749 and the single amino acid mutation L858R in exon $21.1^{10}$ Further clinical trials revealed that the overall survival benefit of afatinib, an oral irreversible ErbB family blocker, was driven mainly by patients with exon 19 positive tumors $(p=0.0001)$ as first-line therapy, whereas in patients with EGFR L858R positive tumors, there was no difference between groups $(p=0.16) .{ }^{11}$ The result indicated that patients with different EGFR mutations show different sensitivities to first-line EGFR-TKIs.

However, studies reported that the significant predictive value of EGFR mutations observed in first-line TKI treatment has not been maintained in second-line TKI treatment. ${ }^{12,13}$ Study also demonstrated that first-line chemotherapy may significantly reduce EGFR mutation rate in NSCLC patients, and the rate of tumor response to second-line TKI therapy was lower than that to first-line therapy in patients with EGFR mutations. ${ }^{14}$ This raises the question: whether different EGFR mutations still show different sensitivities to second-line EGFR-TKIs in the treatment of advanced NSCLC?

Brain and bone metastases were common complications in advanced NSCLC patients, with $30 \%-40 \%$ of patients developed brain and/or bone metastases during the course of disease. ${ }^{15}, 16$ Studies suggested that the prognosis of brain and/or bone metastases patients from NSCLC may be also associated with the status of EGFR mutations. ${ }^{17-20}$ Different EGRF mutations with different EGFR-TKI responses have also been reported in NSCLC patients with brain metastasis. ${ }^{21}$ So, the purpose of this study is to investigate the clinical outcome of NSCLC patients with and without brain and/or bone metastases in different EGFR tumor genotypes (exon
19 deletion vs. exon 21 L858R mutation) receiving second-line EGFR-TKIs.

\section{Materials and Methods}

\section{Patients and clinical characteristics}

Consecutive NSCLC patients harboring either the exon 19 deletion or the L858R point mutation of EGFR treated at the First Affiliated Hospital of Wenzhou Medical University between December 2010 and September 2015 were retrospectively reviewed. The eligibility criteria for this study were as follows: histologically or cytologically confirmed non-small-cell lung cancer; had recurrence or progression after first-line platinum based chemotherapy; with no brain or bone metastasis before the disease progression; received a second-line treatment with gefitinib, icotinib or erlotinib; Eastern Cooperative Oncology Group performance status 0-2 (ECOG PS), with adequate organ functions (including cardiac, hepatic, and renal function) and hematologic functions (absolute neutrophil $\geq 1.5 \times 10^{9} / \mathrm{L}$ or platelet count $\geq 100 \times 10^{9} / \mathrm{L}$ ).

The exclusion criteria were as follows: patients had mixed small cell histology; unknown EGFR mutation status; without at least one measurable lesion according to the Response Evaluation Criteria in Solid Tumors (RECIST) 1.1; lost to follow-up or died within 1 month after the starting of second-line treatment. Patients with brain metastasis who did not receive whole brain radiotherapy (WBRT) or the dose of WBRT less than 30 Gy were also excluded from the subgroup analysis.

The baseline clinical characteristics of enrolled patients were collected through retrospective chart review, which included gender, age at progression, tumor histology, baseline ECOG PS at the start of treatment with second-line EGFR-TKIs, stage of the disease at progression, the type of EGFR-TKIs administered (icotinib, gefitinib or erlotinib).

\section{Treatment and follow-up evaluation}

Oral EGFR-TKIs with gefitinib $(250 \mathrm{mg} / \mathrm{d})$, erlotinib $(150 \mathrm{mg} / \mathrm{d})$ or icotinib $(375 \mathrm{mg} / \mathrm{d})$ were administered to the previously treated NSCLC patients until progression or intolerable adverse effects. WBRT (30 Gy/10F) with concurrently oral EGFR-TKIs were administered for patients with brain metastasis progression. For patients with bone metastasis progression, radiotherapy combining with oral EGFR-TKIs or oral EGFR-TKIs alone was conducted.

Re-evaluation was performed at the beginning of oral EGFR-TKIs treatment and then monthly. Evaluation included physical examination, a complete blood count measurement, liver function test, and 
chest computed tomography (CT) scan. Brain CT with and without contrast, abdominal CT, or bone scan, as well as magnetic resonance images if necessary, were performed when there were relevant symptoms in patients.

\section{Definitions and statistical analyses}

Pearson chi-square or Fisher's exact tests (when there were fewer than 5 expected counts in the contingency table) were applied to compare the baseline characteristics of parents between EGFR genotype groups and to evaluate each potential influential factors. Tumor response to EGFR-TKIs was assessed according to the Response Evaluation Criteria in Solid Tumors 1.1. OS was defined as the interval from the date of first EGFR-TKIs treatment to the date of death. PFS was defined as interval between the date of first EGFR-TKIs treatment and the date of confirming disease progression or death from disease progression. PFS and OS were estimated by using Kaplan-Meier method. Differences between the groups were compared using the log-rank test. The Cox proportional hazard model was used to identify multivariate analyses. The statistical analysis was computed using SPSS (version 17.0, SPSS Inc., Chicago, IL). A p-value $<0.05$ was considered statistically significant.

\section{Results}

As shown in figure 1, of 355 NSCLC patients received EGFR-TKIs as second-line treatment from December 2010 to September 2015, 72 patients due to insufficient information for EGFR mutational analysis or lost to follow-up, 81 patients due to brain and/or bone metastases at their first hospital visit were excluded. Nine patients died within one month after starting the second-line TKIs, 15 had other types of EGFR mutations (not exon 19 and L858R mutation), and 12 patients with brain metastasis without whole brain radiotherapy were also excluded. There were 166 patients enrolled finally.

Table 1 presents the baseline characteristics of enrolled 166 patients with a median age of 60 years (range, 37-87 years). The EGRF mutation types of exon 19 deletion and exon 21 L858R mutation were 51.8\% $(86 / 166)$ and $48.2 \%(80 / 166)$, respectively. Table 2 shows the characteristics of NSCLC patients with brain (75 patients) and bone metastasis (76 patients). The percentage of brain and bone metastases patients with exon 19 deletion and exon 21 L858R mutation were $50.7 \%, 49.3 \%$, and $44.7 \%, 55.3 \%$, respectively. The enrolled patients were well balanced and matched in clinical characteristics.

The responses of all these 166 patients to EGFR-TKIs treatment with a disease control rate
(DCR) of $77.7 \%$ (129/166), and objective response rate (ORR) of $11.4 \%(19 / 166)$, respectively. The ORR and DCR for exon 19 deletion and L858R mutation were $12.8 \%$ vs. $10.0 \% \quad(p=0.61)$, and $81.4 \%$ vs. $73.8 \%$ $(\mathrm{p}=0.64)$, respectively. The overall DCR and ORR of brain and bone metastases were $76 \%$ (57/75), 10.7\% $(8 / 75)$, and $68.4 \%(52 / 76), 3.9 \%$ (3/76), respectively. The ORR and DCR for exon 19 deletion and L858R mutation of brain metastasis patients were $13.2 \%$ vs. $8.1 \% \quad(p=0.48)$, and $78.9 \%$ vs. $73.0 \% \quad(p=0.31)$, respectively. The ORR and DCR for exon 19 deletion and L858R mutation of bone metastasis patients were $5.9 \%$ vs. $2.4 \%(\mathrm{P}=0.44)$, and $70.6 \%$ vs. $66.7 \%(\mathrm{P}=0.72)$, respectively.

Table 1. Baseline characteristics of all 166 non-small-cell lung cancer patients with EGFR exon 19 deletion and L858R mutation.

\begin{tabular}{|c|c|c|c|c|}
\hline Characteristics & Total (\%) & $\begin{array}{l}\text { Exon } 19 \\
\text { deletion }(\%)\end{array}$ & $\begin{array}{l}\text { L858R } \\
\text { mutation (\%) }\end{array}$ & $\mathrm{P}$ \\
\hline All patients & $166(100)$ & $86(100)$ & $80(100)$ & \\
\hline \multicolumn{5}{|l|}{ Gender } \\
\hline Female & $105(63.3)$ & $58(67.4)$ & $47(58.8)$ & \\
\hline Male & $61(36.7)$ & $28(32.6)$ & $33(41.3)$ & 0.57 \\
\hline \multicolumn{5}{|l|}{ Smoking } \\
\hline Never & $91(54.8)$ & $47(54.7)$ & $44(55.0)$ & \\
\hline Current/former & $75(45.2)$ & $39(45.3)$ & $36(45.0)$ & 0.96 \\
\hline \multicolumn{5}{|l|}{ Age at disease progress } \\
\hline$\leq 60$ & $66(38.9)$ & $37(43.0)$ & $29(36.3)$ & \\
\hline$>60$ & $100(60.2)$ & $49(57.0)$ & $51(63.7)$ & 0.37 \\
\hline \multicolumn{5}{|l|}{ Histology } \\
\hline Adenocarcinoma & $153(92.2)$ & $81(94.2)$ & $72(90.0)$ & \\
\hline Non-adenocarcinoma & $13(7.8)$ & $5(5.8)$ & $8(10.0)$ & 0.32 \\
\hline \multicolumn{5}{|c|}{ EGOC PS at disease progress } \\
\hline EGOC 0-1 & $138(83.1)$ & $75(87.2)$ & $63(78.8)$ & \\
\hline EGOC 2-3 & $28(16.9)$ & $11(12.8)$ & $17(21.3)$ & 0.15 \\
\hline \multicolumn{5}{|l|}{$\begin{array}{l}\text { Stage at disease } \\
\text { progress }\end{array}$} \\
\hline III & $9(5.4 \%)$ & $6(7.0)$ & $3(3.8)$ & \\
\hline IV & $157(94.6)$ & $80(93.0)$ & $77(96.3)$ & 0.36 \\
\hline \multicolumn{5}{|l|}{ Type of EGFR-TKIs } \\
\hline Erlotinib & $41(24.7)$ & $16(18.6)$ & $25(31.3)$ & \\
\hline Gefitinib & $72(43.4)$ & $45(52.3)$ & $27(33.8)$ & \\
\hline Icotinib & $53(31.9)$ & $25(29.1)$ & $28(35.0)$ & 0.21 \\
\hline \multicolumn{5}{|c|}{ Control by first-line chemotherapy } \\
\hline Control & $139(83.7)$ & $74(86.0)$ & $65(81.3)$ & \\
\hline $\mathrm{NO}$ & $27(16.3)$ & $12(14.0)$ & $15(18.8)$ & 0.4 \\
\hline \multicolumn{5}{|l|}{ Brain metastasis } \\
\hline No & $91(54.8)$ & $48(55.8)$ & $43(53.8)$ & \\
\hline Yes & $75(45.2)$ & $38(44.2)$ & $37(46.3)$ & 0.79 \\
\hline \multicolumn{5}{|l|}{ Bone metastasis } \\
\hline No & $90(54.2)$ & $52(60.5)$ & $38(47.5)$ & \\
\hline Yes & $76(45.8)$ & $34(39.5)$ & $42(52.5)$ & 0.09 \\
\hline \multicolumn{5}{|l|}{$\begin{array}{l}\text { Subsequent systemic } \\
\text { therapy }\end{array}$} \\
\hline No & $50(30.1)$ & $24(27.9)$ & $26(32.5)$ & \\
\hline Yes & $116(69.9)$ & $62(72.1)$ & $54(67.5)$ & 0.52 \\
\hline
\end{tabular}

Abbreviations: EGFR, epidermal growth factor receptor; TKIs, tyrosine kinase inhibitors; ECOG PS, Eastern Cooperative Oncology Group performance status. 


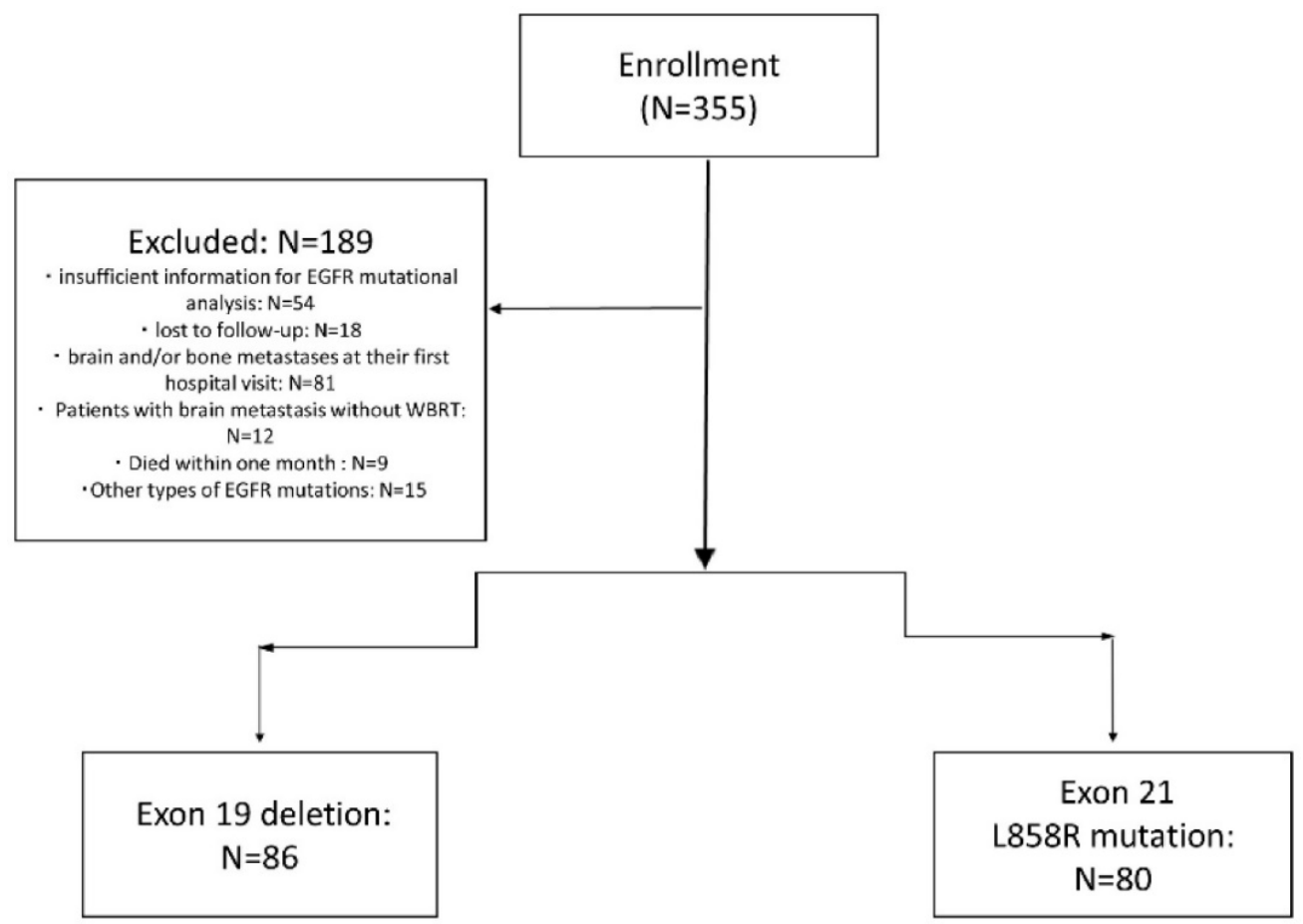

Figure 1. Flow Diagram of all patients.

Table 2. Baseline characteristics of non-small-cell lung cancer patients with brain and bone metastases with EGFR exon 19 deletion and L858R mutation.

\begin{tabular}{|c|c|c|c|c|c|c|c|c|}
\hline \multirow[b]{2}{*}{ Characteristics } & \multicolumn{4}{|c|}{ Brain metastasis } & \multicolumn{4}{|c|}{ Bone metastasis } \\
\hline & Number & Exon $19(\%)$ & Exon $21(\%)$ & $\mathrm{p}$ & Number & Exon $19(\%)$ & Exon $21(\%)$ & $\mathrm{p}$ \\
\hline Total patients & $75(100)$ & $38(50.7)$ & $37(49.3)$ & & $76(100)$ & $34(44.7)$ & $42(55.3)$ & \\
\hline \multicolumn{9}{|l|}{ Gender } \\
\hline Female & $47(62.7 \%)$ & $26(68.4)$ & $21(56.8)$ & & $48(63.2)$ & $24(70.6)$ & $24(57.1)$ & \\
\hline Male & $28(37.3)$ & $12(31.6)$ & $16(43.2)$ & 0.3 & $28(36.8)$ & $10(29.4)$ & $18(42.9)$ & 0.2 \\
\hline \multicolumn{9}{|l|}{ Smoking } \\
\hline Never & $44(58.7)$ & $23(60.5)$ & $21(56.8)$ & & $45(59.2)$ & $22(64.7)$ & $23(54.8)$ & \\
\hline Current/former & $31(41.3)$ & $15(39.5)$ & $16(43.2)$ & 0.74 & $31(40.8)$ & $12(35.3)$ & $19(45.2)$ & 0.4 \\
\hline \multicolumn{9}{|l|}{ Age at disease progress } \\
\hline$\leq 60$ & $31(41.3)$ & $19(50.0)$ & $12(32.4)$ & & $28(36.8)$ & $14(41.2)$ & $14(33.3)$ & \\
\hline$>60$ & $44(58.7)$ & $19(50.0)$ & $25(67.6)$ & 0.12 & $48(63.2)$ & $20(58.8)$ & $28(66.7)$ & 0.5 \\
\hline \multicolumn{9}{|l|}{ Histology } \\
\hline Adenocarcinoma & $71(94.7)$ & $36(94.7)$ & $35(94.6)$ & & $69(90.8)$ & 33(97.1) & $36(85.7)$ & \\
\hline Non-adenocarcinoma & $4(5.3)$ & $2(5.3)$ & $2(5.4)$ & 0.98 & $7(9.2 \%)$ & $1(2.9)$ & $6(14.3)$ & 0.1 \\
\hline \multicolumn{9}{|c|}{ EGOC PS at disease progress } \\
\hline EGOC 0-1 & $60(80.0)$ & $31(81.6)$ & $29(78.4)$ & & $65(85.5)$ & $29(85.3)$ & $36(85.7)$ & \\
\hline EGOC 2-3 & $15(20.0)$ & $7(18.4)$ & $8(21.6)$ & 0.77 & $11(14.5)$ & $5(14.7)$ & $6(14.3)$ & 1 \\
\hline \multicolumn{9}{|l|}{ Type of EGFR-TKI } \\
\hline Erlotinib & $18(24.0)$ & $8(21.1)$ & $10(27.0)$ & & $16(21.1)$ & $4(11.8)$ & $12(28.6)$ & \\
\hline Gefitinib & $30(40.0)$ & $17(44.7)$ & $13(35.1)$ & & $39(51.3)$ & $22(64.7)$ & $17(40.5)$ & \\
\hline Icotinib & $27(36.0)$ & $13(34.2)$ & $14(37.8)$ & 0.68 & $21(27.6)$ & $8(23.5)$ & $13(31.0)$ & 0.1 \\
\hline \multicolumn{9}{|c|}{ Control by first-line chemotherapy } \\
\hline Control & $61(81.3)$ & $31(81.6)$ & $30(81.1)$ & & $65(85.5)$ & $30(88.2)$ & $35(83.3)$ & \\
\hline No & $14(18.7)$ & $7(18.4)$ & $7(18.9)$ & 0.96 & $11(14.5)$ & $4(11.8)$ & $7(16.7)$ & 0.6 \\
\hline \multicolumn{9}{|l|}{ Number of BM } \\
\hline 1 & $21(28.0)$ & $12(31.6)$ & $9(24.3)$ & & 15(19.7) & $7(20.6)$ & $8(19.0)$ & \\
\hline$>1$ & $54(72.0)$ & $26(68.4)$ & $28(75.7)$ & 0.48 & $61(80.3)$ & $27(79.4)$ & $34(81.0)$ & 0.9 \\
\hline \multicolumn{9}{|l|}{ Extracranial metastases } \\
\hline No & $27(36.0)$ & 12(31.6) & $15(40.5)$ & & $39(51.3)$ & $18(52.9)$ & $21(50.0)$ & \\
\hline Yes & $48(64.0)$ & $26(68.4)$ & $22(59.5)$ & 0.42 & $37(48.7)$ & $16(47.1)$ & $21(50.0)$ & 0.8 \\
\hline \multicolumn{9}{|c|}{ Subsequent systemic therapy } \\
\hline No & 19(33.9) & $12(31.6)$ & $7(18.9)$ & & $24(31.6)$ & $11(32.4)$ & $13(31.0)$ & \\
\hline Yes & $56(66.1)$ & $26(68.4)$ & $30(81.1)$ & 0.21 & $52(68.4)$ & $23(67.6)$ & $29(69.0)$ & 0.9 \\
\hline
\end{tabular}

Abbreviations: EGFR, epidermal growth factor receptor; TKIs, tyrosine kinase inhibitors; ECOG PS, Eastern Cooperative Oncology Group performance status; 
The median progression-free survival (PFS) and overall survival (OS) of enrolled NSCLC patients was 5.8 months (95\% Cl, 4.9-6.7 months) and 12.4 months (95\% Cl, 10.6-14.1 months), respectively. The estimated 6-month and 1-year PFS rates were $57.6 \%$, $13.1 \%$ in the exon 19 deletion arm, and $38.8 \%, 6.7 \%$ in the L858R mutation arm, respectively. Figure 2 presents the median PFS and OS of NSCLC patients after second-line EGFR-TKIs treatment for exon 19 deletion and L858R mutation. The exon 19 deletion group had a significantly longer median PFS compared with the L858R mutation group (6.7 vs. 4.5 months, $\mathrm{P}=0.002$ ) as shown in figure $2 \mathrm{~A}$. The estimated 6-month and 1-year OS rates were $97.4 \%$, $61.6 \%$, and $87.3 \%, 46.6 \%$ for exon 19 deletion and L858R mutation group, respectively. The exon 19 deletion group had a longer median OS compared with the L858R mutation group (13.7 vs. 11.7 months, $\mathrm{P}=0.02)$, as shown in figure $2 \mathrm{~B}$.

The median PFS and OS were 4.7 months (95\% $\mathrm{Cl}$, 3.9-5.5 months) and 10.3 months $(95 \% \mathrm{Cl}, 8.2-12.5$ months) for NSCLC patients with brain metastasis, respectively. As shown in figure 3A, the probabilities of PFS at 6 months and 1 year were $61.9 \%$ and $12.9 \%$ in the exon 19 deletion, $20 \%$ and $0 \%$ in L858R mutation arms, respectively. Exon 19 deletion group had a longer median PFS than the L858R mutation group (6.7 vs. 3.9 months, $p<0.001)$. The OS was shown in figure $3 \mathrm{~B}$, in which the probabilities of OS at 6 months and 1 year were $93.8 \%$ and $54.5 \%$ for the exon 19 deletion arm, and $81.6 \%$ and $18.5 \%$ for L858R mutation arm, respectively. The exon 19 deletion arm had a longer median OS compared with the L858R mutation arm (13.7 vs. 7.9 months, $\mathrm{p}=0.006)$.

The median PFS and OS for NSCLC patients with bone metastasis were 4.8 months $(95 \% \mathrm{Cl}, 4.0-5.6$ months) and 12.9 months $(95 \% \mathrm{Cl}, 8.7-17.1$ months), respectively. The estimated 6-month and 1-year DFS rates for exon 19 deletion and the L858R mutation groups were $46.9 \%, 16.3 \%$, and $31.0 \%, 10.7 \%$, respectively. There was no significant difference on median PFS (5.9 vs. 4.4 months; $\mathrm{P}=0.17$ ) and median OS between patients with exon 19 deletion and L858R mutation, as shown in figure 4B. The estimated 6-month and 1-year OS rates were $96.0 \%, 63.1 \%$, and $91.9 \%, 45.6 \%$ for patients with exon 19 deletion and L858R mutation, respectively.

Univariate and multivariate analysis on PFS and OS for all NSCLC patients and for patients with brain and bone metastases were shown in table 3. EGFR genotype and ECOG PS were independent predictors of PFS for both NSCLC patients with and without brain metastasis. Never smoking $(\mathrm{P}=0.001)$, exon 19 deletion $(\mathrm{P}=0.03)$, EGOC PS $(0-1)(\mathrm{P}<0.001)$ and no brain metastasis $(\mathrm{p}=0.01)$ were correlated with longer OS for all NSCLC patients. For patients with brain metastasis, age at disease progression $(p=0.009)$, genotype $(p=0.02)$ and EGOC PS $(p<0.001)$ were independent predictors of OS. For patients with bone metastasis, EGOC PS $(p<0.001)$ was an independent predictor for both PFS and OS. Female $(\mathrm{P}=0.01)$, never smoking $(\mathrm{P}=0.005)$, number of bone metastases $(\mathrm{P}=0.03)$ and EGOC PS $(0-1)(\mathrm{P}=0.002)$ were related to a longer PFS. EGOC PS $(0-1)(P<0.001)$ was associated with a longer OS.

Table 3. Multivariate analysis results for progression-free survival and overall survival in non-small-cell lung cancer patients according to clinicopathologic characteristics.

\begin{tabular}{|c|c|c|c|c|c|c|c|c|c|}
\hline & \multirow[t]{3}{*}{$\mathrm{N}$} & \multicolumn{5}{|l|}{ PFS } & \multicolumn{3}{|l|}{ OS } \\
\hline & & \multirow{2}{*}{$\begin{array}{l}\text { Univariate } \\
\mathrm{p}\end{array}$} & \multicolumn{3}{|c|}{ Multivariate } & \multirow{2}{*}{$\begin{array}{l}\text { Univariate } \\
\mathrm{p}\end{array}$} & \multicolumn{3}{|c|}{ Multivariate } \\
\hline & & & HR & $95 \% \mathrm{CI}$ & $\mathrm{p}$ & & HR & $95 \% \mathrm{CI}$ & $\mathrm{p}$ \\
\hline \multicolumn{10}{|l|}{ All NSCLC patients } \\
\hline Gender (Female/Male) & $104 / 62$ & 0.03 & 1.25 & $0.87-1.82$ & 0.23 & 0.44 & & & \\
\hline Smoking (Never / Current or former) & $91 / 75$ & 0.35 & & & & 0.02 & 2.34 & $1.33-4.09$ & 0.003 \\
\hline Age at disease progression $(\leq 60 />60)$ & $66 / 100$ & 0.20 & & & & 0.07 & 1.72 & $0.94-3.15$ & 0.08 \\
\hline $\begin{array}{l}\text { EGOC PS at disease progression } \\
\text { (EGOC } 0-1 / 2-3)\end{array}$ & $138 / 28$ & $<0.001$ & 3.67 & 2.24-6.01 & $<0.001$ & $<0.001$ & 11.86 & $5.55-25.36$ & $<0.001$ \\
\hline Genotype (exon 19/21) & $86 / 80$ & 0.002 & 1.54 & $1.08-2.20$ & 0.02 & 0.02 & 1.94 & $1.09-3.48$ & 0.03 \\
\hline Brain metastases (No/Yes) & $91 / 75$ & 0.07 & & & & 0.02 & 2.02 & $1.17-3.50$ & 0.01 \\
\hline \multicolumn{10}{|l|}{ Patients with brain metastasis } \\
\hline Age at disease progression $(\leq 60 />60)$ & $31 / 44$ & 0.34 & & & & 0.004 & 3.38 & $1.35-8.47$ & 0.009 \\
\hline $\begin{array}{l}\text { EGOC PS at disease progression (EGOC } \\
0-1 / 2-3 \text { ) }\end{array}$ & C $60 / 15$ & $<0.001$ & 3.1 & $1.58-6.05$ & 0.001 & $<0.001$ & 5.59 & $2.34-13.36$ & $<0.001$ \\
\hline Genotype (exon 19/21) & $38 / 37$ & $<0.001$ & 2.60 & $1.51-4.48$ & 0.001 & 0.006 & 2.67 & $1.17-6.05$ & 0.02 \\
\hline \multicolumn{10}{|l|}{ Patients with bone metastasis } \\
\hline Gender (Female/Male) & $48 / 28$ & 0.01 & 2.07 & $1.16-3.73$ & 0.02 & 0.80 & & & \\
\hline Smoking (Never / Current or former) & $45 / 31$ & 0.005 & 1.75 & $0.96-3.19$ & 0.07 & 0.02 & 1.99 & $0.77-5.13$ & 0.16 \\
\hline $\begin{array}{l}\text { EGOC PS at disease progression (EGOC } \\
0-1 / 2-3 \text { ) }\end{array}$ & C $65 / 11$ & 0.002 & 2.83 & $1.30-6.19$ & 0.009 & $<0.001$ & 7.79 & $2.52-24.09$ & $<0.001$ \\
\hline Number of bone metastasis $(<1 />1)$ & $15 / 61$ & 0.03 & 1.69 & $0.82-3.50$ & 0.16 & 0.004 & 4.29 & $0.850-21.72$ & 0.08 \\
\hline
\end{tabular}



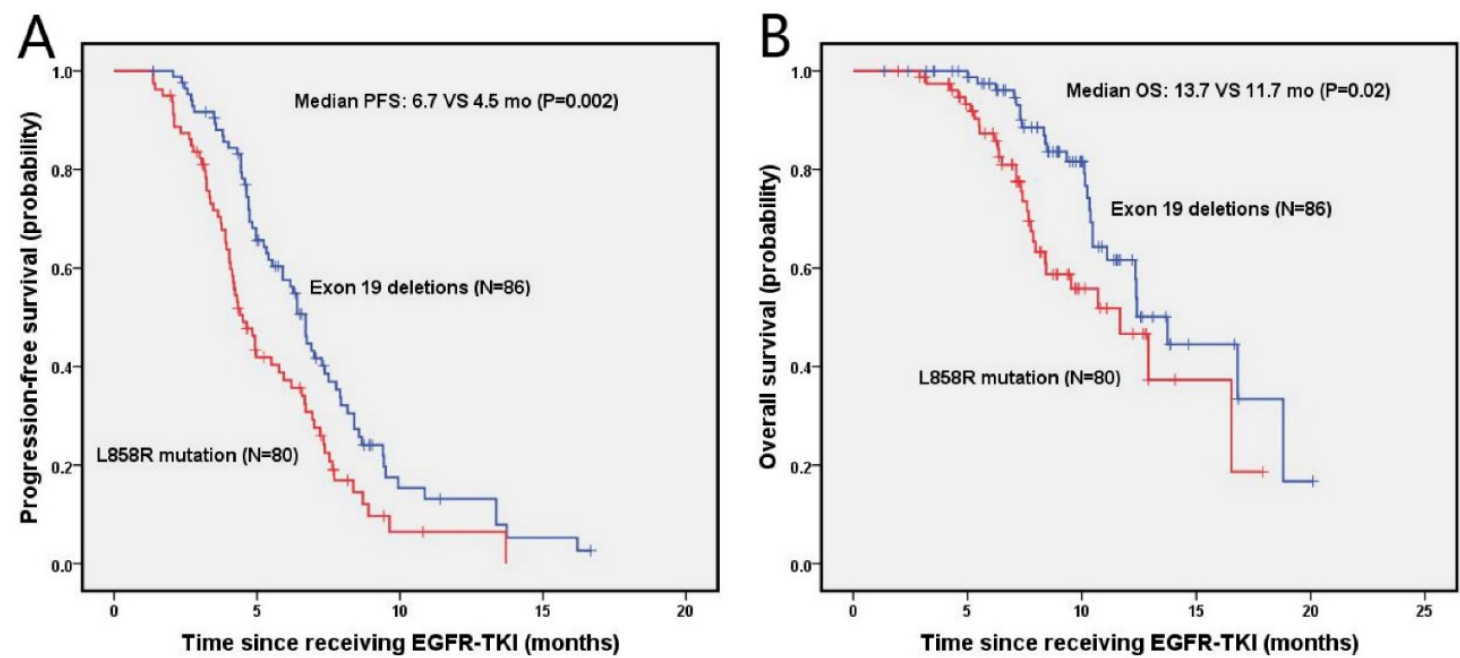

Figure 2. Kaplan-Meier PFS (A) and OS (B) curves for the all NSCLC patients between exon 19 and with 21 mutation. Abbreviations: PFS, The disease-free survival; OS: the overall survival; NSCLC: non-small-cell lung cancer.


Figure 3. Kaplan-Meier PFS (A) and OS (B) curves for the NSCLC patients with brain metastasis between exon 19 and with 21 mutation. Abbreviations: PFS, The disease-free survival; OS: the overall survival; NSCLC: non-small-cell lung cancer.


Figure 4. Kaplan-Meier PFS (A) and OS (B) curves for the NSCLC patients with bone metastasis between exon 19 and with 21 mutation. Abbreviations: PFS, The disease-free survival; OS: the overall survival; NSCLC: non-small-cell lung cancer. 
Rash (47/166), fatigue (46/166), and anorexia $(25 / 166)$ were the three most frequent side effects observed for NSCLC patients treated with second-line EGFR-TKIs. The common toxicities of grade III/IV were rash (9/166) and fatigue (7/166). Most patients tolerated well with the side effects after symptomatic treatments, except for a dose reduction of EGFR-TKIs for 15 patients. There was no significant difference on side effects between NSCLC patients with exon 19 and 21 mutations.

\section{Discussion}

The efficacy of second-line EGFR-TKIs in the treatment of advanced NSCLC patients with and without brain and bone metastases, and the effects of different genotype mutations on PFS and OS were investigated in this study. Advanced NSCLC patients with and without brain metastasis with an exon 19 deletion showed a better PFS and OS compared with those with L858R mutation receiving second-line EGFR-TKIs treatment. No significant difference on PFS and OS between exon 19 and 21 mutations was observed for patients with bone metastasis.

Studies demonstrated that advanced NSCLC patients underwent first-line platinum-based doublet chemotherapy need EGFR-TKIs as second-line or third-line treatment to maximize the survival benefit. 22 The ORR of our patients was $11.4 \%$, which was similar to the reported $11.3 \%$ from a phase II study on the EGFR wild type NSCLC patients treated with second-line EGFR-TKIs, while the DCR in this study was much higher $(76.5 \%$ vs. $28.3 \%) .{ }^{23}$ This may be due to the intrinsic sensitivity differences for different type of EGFR mutations. The ORR and DCR in our study were a bit inferior to the reported $26.7 \%$ and $81.6 \%$ in the TRUST study with Chinese subpopulation. ${ }^{24}$ However, no specific EGFR mutation types was reported, and the results of second-line and third-line treatment were mixed in that study. The median PFS in our study was close to that of TRUST study with Chinese subpopulation (5.8 vs. 6.4 months). The median OS in our study was 12.4 months (95\% Cl, 10.6-14.1 months), which was close to the reported 11.5 months in a phase III study and 13.3 months in a phase II study using TKIs as a second-line in the treatment of advanced NSCLC, ${ }^{25,26}$ but much shorter than reported 27 months in the study of Rosell $\mathrm{R}$ et al. ${ }^{27}$ The difference may be due to a higher percentage $(45.2 \%)$ of NSCLC patients with brain metastasis were enrolled in our study, and due to other patients' characteristics discrepancies in different studies.

It has been reported that more than $25 \%$ patients with lung cancer developed brain metastasis during the disease courses despite systemic treatment and/or local radiotherapy. ${ }^{28,}{ }^{29}$ There was limited data for the response of brain metastasis to EGFR-TKIs. Recently, Park et al reported a median PFS and OS of 6.6 months (95\% CI, 3.8-9.3 months) and 15.9 months (95\% CI, 7.2-24.6 months), respectively, in a phase II study in NSCLC patients with brain metastasis. ${ }^{30}$ These results of first-line EGFR-TKIs treatment were better than the results of our second-line TKIs treatment on brain metastasis (4.7 and 10.3 months for PFS and OS, respectively). Next to brain, bone is a frequent site of metastasis in NSCLC exerting a negative impact on quality of life. ${ }^{31,32}$ Bone metastasis was an independent negative predictive factor for OS in patients with mutated and wild-type EGFR. ${ }^{33}$ In this study, we achieved a median PFS and OS of 4.8 and 12.9 months for NSCLC patients with bone metastasis, respectively. The median OS was longer than reported 8 months of bone metastasis NSCLC with wild-type EGFR. ${ }^{34}$

Several previous studies had investigated the relationship between EGFR mutation status and prognosis of NSCLC patients. Some studies demonstrated the superiority of exon 19 deletion over exon 21 point mutation in NSCLC patients treated with erlotinib and/or gefitinib,27, 35, 36 while other studies did not. ${ }^{37}$, 38 The NCT00344773 study demonstrated that ORR and DCR were higher in patients with exon 19 deletion than those with L858R mutation (ORR: $62.1 \%$ vs $33.3 \%$; $\mathrm{P}=0.07$; DCR: $96.6 \%$ vs $66.7 \% ; \mathrm{p}=0.006){ }^{39}$ Sun $\mathrm{JM}$ et al reported that patients with exon 19 deletion $(\mathrm{n}=58)$ had a significantly longer PFS than patients with L858R mutation $(n=19)(9.5$ vs. 7.7 months; $\mathrm{p}=0.03)$ in the treatment of 77 patients with EGFR-TKI. ${ }^{40}$ While, Igawa $S$ et al demonstrated that there was no significant difference between patients with the exon 19 deletion and L858R point mutation on ORR $(p=0.44)$, PFS $(p=0.81)$ and OS $(p=0.95)$ in a study of 124 NSCLC patients harboring active EGFR mutations (exon 19 deletion: 68 patients, L858R mutation: 56 patients). ${ }^{41}$

Studies also demonstrated that the significant predictive value of EGFR mutations observed in first-line TKI treatment has not been maintained in second-line TKI treatment ${ }^{14}$. According to a randomized phase III trial, the qualitative difference in response seen in the first line was not demonstrated in second-line settings. ${ }^{42}$ In this study, although no significant difference on ORR and DCR between exon 19 deletion and L858R mutation groups were observed, the exon 19 deletion arm showed a significant higher PFS and OS than L858R mutation arm in advanced NSCLC patient with and without brain metastasis receiving second-line EGFR-TKIs. This was similar to the results of a second-line TKI 
treatment with erlotinib for patients with brain metastasis, in which patients with exon 19 deletion showed longer OS than those with exon 21 point mutation $(p=0.02)$. ${ }^{21}$ This indicated that EGFR mutations are good prognostic factors, as well as have potential significant predictive value, for advanced NSCLC patients receiving second-line TKIs.

In this study, multivariate analysis indicated that EGFR genotype and ECOG PS were independent predictors of PFS for both NSCLC patients with and without brain metastasis. Never smoking status ( $\mathrm{P}=0.001)$, exon 19 deletion $(\mathrm{P}=0.03)$, EGOC PS (0-1) $(\mathrm{P}<0.001)$ and no brain metastasis $(\mathrm{p}=0.01)$ were correlated with longer OS for all NSCLC patients. For patients with brain metastasis, age at disease progression $(p=0.009)$, genotype $(p=0.02)$ and EGOC PS $(p<0.001)$ were independent predictors of OS. Similarly, studies reported that the favorable prognostic factors for NSCLC patients with brain metastasis were EGFR mutations, stable extracranial disease at the time of brain metastasis diagnosis, and EGOC PS. ${ }^{33,44}$ WBRT has been regarded as the standard treatment for brain metastases and applied to all the patients with brain metastasis in this study. ${ }^{30}$ Reports demonstrated a favorable clinical effect of the combination of WBRT and gefitinib or erlotinib therapy with an excellent intracranial disease control rate, which suggested that WBRT might serve as some form of sensitizer for EGFR-TKI therapy. ${ }^{21}$

For NSCLC patients with bone metastasis in this study, no-smoking $(\mathrm{P}=0.005)$, number of bone metastases ( $\mathrm{P}=0.03)$ and ECOG PS (0-1) $(\mathrm{P}=0.002)$ were related to a longer PFS. EGOC PS $(0-1)(\mathrm{P}<0.001)$ was associated with a longer OS. However, EGFR mutations were not significant predictors for NSCLC patients with bone metastasis, although it had been reported in previous study that epidermal growth factor (EGF) signaling was an important mediator of bone metastasis in many cancers, as well as in NSCLC patients. 45,46

One limitation of current study is that it is a retrospective methodology from a single-institution experience. The impact of various treatments related outcome could not be fully evaluated. The number of patients enrolled may be not sufficient enough and the follow-up duration of the study may be not long enough. External validation by using other large database for evaluating the prognostic effect second-line EGFR-TKIs in the treatment of NSCLC would be of value to further explore the benefit of EGFR mutations.

In a conclusion, with the advancements in the treatment of NSCLC, many patients may be candidates for further systemic therapy using chemotherapy and/or EGFR-TKIs as second-line and third-line treatments. Our study demonstrated NSCLC patients harboring exon 19 deletion achieved better PFS and OS than those with L858R mutation, indicating that EGFR mutations are significant prognostic predictors for advanced NSCLC patients with and without brain metastasis receiving second-line EGFR-TKIs treatment.

\section{Abbreviations}

EGFR: epidermal growth factor receptor; NSCLC: non-small-cell lung cancer; TKIs: tyrosine kinase inhibitors; DCR: disease control rate; ORR: objective response rate; PFS: The disease-free survival; OS: the overall survival; ECOG PS: Eastern Cooperative Oncology Group performance status; RCTs: randomized-controlled trials; WBRT: whole brain radiotherapy; CT: computed tomography; EGF: epidermal growth factor.

\section{Acknowledgments}

The study was partially supported grants from the Wenzhou Municipal Science and Technology Bureau (H20100068), (Y20140709), the Natural Science Foundation of Zhejiang Province (LY16H160047) and National Natural Science Foundation of China (11675122).

\section{Ethics Committee Approval and Patient Consent}

All procedures performed in studies involving human participants were in accordance with the ethical standards of the institutional and/or national research committee and with the 1964 Declaration of Helsinki and its later amendments or comparable ethical standards. For this type of study, formal consent is not required. This article does not contain any studies with animals performed by any of the authors.

\section{Competing Interests}

The authors have declared that no competing interest exists.

\section{References}

1. Chen W, Zhang S, Zou X. [Estimation and projection of lung cancer incidence and mortality in China]. Zhongguo fei ai za zhi=Chinese journal of lung cancer 2010;13:488-493.

2. Steuer CE, Ramalingam SS. Targeting EGFR in lung cancer: Lessons learned and future perspectives. Molecular aspects of medicine 2015

3. Govindan R, Page N, Morgensztern D, et al. Changing epidemiology of small-cell lung cancer in the United States over the last 30 years: analysis of the surveillance, epidemiologic, and end results database. Journal of clinical oncology : official journal of the American Society of Clinical Oncology 2006;24:4539-4544.

4. Chen Z, Zhong B, Lun X, et al. Specific Safety Profile of Bevacizumab in Asian Patients With Advanced NSCLC: A Meta-Analysis. Medicine 2015;94:e975.

5. Siegel R, DeSantis C, Virgo K, et al. Cancer treatment and survivorship statistics, 2012. CA: a cancer journal for clinicians 2012;62:220-241.

6. Yang $\mathrm{CH}, \mathrm{Yu} \mathrm{CJ}$, Shih JY, et al. Specific EGFR mutations predict treatment outcome of stage IIIB/IV patients with chemotherapy-naive non-small-cell lung cancer receiving first-line gefitinib monotherapy. Journal of clinical 
oncology : official journal of the American Society of Clinical Oncology 2008;26:2745-2753.

7. Balak MN, Gong Y, Riely GJ, et al. Novel D761Y and common secondary T790M mutations in epidermal growth factor receptor-mutant lung adenocarcinomas with acquired resistance to kinase inhibitors. Clinical cancer research : an official journal of the American Association for Cancer Research 2006;12:6494-6501.

8. Wu JY, Wu SG, Yang $\mathrm{CH}$, et al. Lung cancer with epidermal growth factor receptor exon 20 mutations is associated with poor gefitinib treatment response. Clinical cancer research : an official journal of the American Association for Cancer Research 2008;14:4877-4882

9. Wu JY, Yu CJ, Chang YC, et al. Effectiveness of tyrosine kinase inhibitors on "uncommon" epidermal growth factor receptor mutations of unknown clinical significance in non-small cell lung cancer. Clinical cancer research : an official journal of the American Association for Cancer Research 2011;17:3812-3821.

10. Gazdar AF. Activating and resistance mutations of EGFR in non-small-cell lung cancer: role in clinical response to EGFR tyrosine kinase inhibitors. Oncogene 2009;28 Suppl 1:S24-31.

11. Yang JC, $\mathrm{Wu} \mathrm{YL}$, Schuler $\mathrm{M}$, et al. Afatinib versus cisplatin-based chemotherapy for EGFR mutation-positive lung adenocarcinoma (LUX-Lung 3 and LUX-Lung 6): analysis of overall survival data from two randomised, phase 3 trials. The Lancet Oncology 2015;16:141-151.

12. Shepherd FA, Rodrigues Pereira J, Ciuleanu T, et al. Erlotinib in previously treated non-small-cell lung cancer. The New England journal of medicine 2005;353:123-132.

13. Thatcher N, Chang A, Parikh P, et al. Gefitinib plus best supportive care in previously treated patients with refractory advanced non-small-cell lung cancer: results from a randomised, placebo-controlled, multicentre study (Iressa Survival Evaluation in Lung Cancer). Lancet 2005;366:1527-1537.

14. Bai H, Wang $Z$, Chen $K$, et al. Influence of chemotherapy on EGFR mutation status among patients with non-small-cell lung cancer. Journal of clinical oncology : official journal of the American Society of Clinical Oncology 2012;30:3077-3083.

15. Rosen LS, Gordon D, Tchekmedyian NS, et al. Long-term efficacy and safety of zoledronic acid in the treatment of skeletal metastases in patients with nonsmall cell lung carcinoma and other solid tumors: a randomized, Phase III, double-blind, placebo-controlled trial. Cancer 2004;100:2613-2621.

16. Huang $Q$, Ouyang X. Predictive biochemical-markers for the development of brain metastases from lung cancer: clinical evidence and future directions. Cancer epidemiology 2013;37:703-707.

17. Zukawa M, Nakano M, Hirano N, et al. The effectiveness of gefitinib on spinal metastases of lung cancer - report of two cases. Asian spine journal 2008;2:109-113.

18. Wu YL, Zhou C, Cheng $\mathrm{Y}$, et al. Erlotinib as second-line treatment in patients with advanced non-small-cell lung cancer and asymptomatic brain metastases: a phase II study (CTONG-0803). Annals of oncology : official journal of the European Society for Medical Oncology / ESMO 2013;24:993-999.

19. Fan $Y, X u X, X i e ~ C$. EGFR-TKI therapy for patients with brain metastases from non-small-cell lung cancer: a pooled analysis of published data. OncoTargets and therapy 2014;7:2075-2084.

20. Welsh JW, Komaki R, Amini A, et al. Phase II trial of erlotinib plus concurrent whole-brain radiation therapy for patients with brain metastases from non-small-cell lung cancer. Journal of clinical oncology : official journal of the American Society of Clinical Oncology 2013;31:895-902.

21. Sekine A, Satoh $\mathrm{H}$, Iwasawa $\mathrm{T}$, et al. Prognostic factors for brain metastases from non-small cell lung cancer with EGFR mutation: influence of stable extracranial disease and erlotinib therapy. Medical oncology 2014;31:228.

22. Zhang JW, Zhao YY, Guo Y, et al. The impact of both platinum-based chemotherapy and EGFR-TKIs on overall survival of patients with advanced non-small cell lung cancer. Chinese journal of cancer 2014;33:105-114.

23. Morise M, Taniguchi H, Saka $\mathrm{H}$, et al. Phase II study of erlotinib for previously treated patients with EGFR wild-type non-small-cell lung cancer, following EGFR mutation status reevaluation with the Scorpion Amplified Refractory Mutation System. Molecular and clinical oncology 2014;2:991-996.

24. Huang $Y$, Zhang L, Shi $\mathrm{Y}$, et al. Efficacy of erlotinib in previously treated patients with advanced non-small cell lung cancer: analysis of the Chinese subpopulation in the TRUST study. Japanese journal of clinical oncology 2015;45:569-575.

25. Maruyama $R$, Nishiwaki $Y$, Tamura $T$, et al. Phase III study, V-15-32, of gefitinib versus docetaxel in previously treated Japanese patients with non-small-cell lung cancer. Journal of clinical oncology : official journal of the American Society of Clinical Oncology 2008;26:4244-4252.

26. Chen YM, Liu JM, Chou TY, et al. Phase II randomized study of daily gefitinib treatment alone or with vinorelbine every 2 weeks in patients with adenocarcinoma of the lung who failed at least 2 regimens of chemotherapy. Cancer 2007:109:1821-1828.

27. Rosell R, Moran T, Queralt C, et al. Screening for epidermal growth factor receptor mutations in lung cancer. The New England journal of medicine 2009;361:958-967.

28. Mehta MP, Rodrigus $\mathrm{P}$, Terhaard $\mathrm{CH}$, et al. Survival and neurologic outcomes in a randomized trial of motexafin gadolinium and whole-brain radiation therapy in brain metastases. Journal of clinical oncology : official journal of the American Society of Clinical Oncology 2003;21:2529-2536.
29. Burel-Vandenbos F, Ambrosetti D, Coutts M, et al. EGFR mutation status in brain metastases of non-small cell lung carcinoma. Journal of neuro-oncology 2013;111:1-10.

30. Park SJ, Kim HT, Lee DH, et al. Efficacy of epidermal growth factor receptor tyrosine kinase inhibitors for brain metastasis in non-small cell lung cancer patients harboring either exon 19 or 21 mutation. Lung cancer 2012;77:556-560.

31. Langer CJ, Mehta MP. Current management of brain metastases, with a focus on systemic options. Journal of clinical oncology : official journal of the American Society of Clinical Oncology 2005;23:6207-6219.

32. Langer C, Hirsh V. Skeletal morbidity in lung cancer patients with bone metastases: demonstrating the need for early diagnosis and treatment with bisphosphonates. Lung cancer 2010;67:4-11.

33. Fujitani K, Tamura S, Kimura Y, et al. Three-year outcomes of a phase II study of adjuvant chemotherapy with S-1 plus docetaxel for stage III gastric cancer after curative D2 gastrectomy. Gastric cancer : official journal of the International Gastric Cancer Association and the Japanese Gastric Cancer Association 2014;17:348-353.

34. Scagliotti GV, Hirsh V, Siena S, et al. Overall survival improvement in patients with lung cancer and bone metastases treated with denosumab versus zoledronic acid: subgroup analysis from a randomized phase 3 study. Journal of thoracic oncology : official publication of the International Association for the Study of Lung Cancer 2012;7:1823-1829.

35. Won YW, Han JY, Lee GK, et al. Comparison of clinical outcome of patients with non-small-cell lung cancer harbouring epidermal growth factor receptor exon 19 or exon 21 mutations. Journal of clinical pathology 2011;64:947-952.

36. Heon S, Yeap BY, Britt GJ, et al. Development of central nervous system metastases in patients with advanced non-small cell lung cancer and somatic EGFR mutations treated with gefitinib or erlotinib. Clinical cancer research: an official journal of the American Association for Cancer Research 2010;16:5873-5882.

37. Maemondo M, Inoue A, Kobayashi K, et al. Gefitinib or chemotherapy for non-small-cell lung cancer with mutated EGFR. The New England journal of medicine 2010;362:2380-2388.

38. Mitsudomi T, Morita S, Yatabe Y, et al. Gefitinib versus cisplatin plus docetaxel in patients with non-small-cell lung cancer harbouring mutations of the epidermal growth factor receptor (WJTOG3405): an open label, randomised phase 3 trial. The Lancet Oncology 2010;11:121-128.

39. Kim DW, Lee SH, Lee JS, et al. A multicenter phase II study to evaluate the efficacy and safety of gefitinib as first-line treatment for Korean patients with advanced pulmonary adenocarcinoma harboring EGFR mutations. Lung cancer 2011;71:65-69.

40. Sun JM, Won YW, Kim ST, et al. The different efficacy of gefitinib or erlotinib according to epidermal growth factor receptor exon 19 and exon 21 mutations in Korean non-small cell lung cancer patients. Journal of cancer research and clinical oncology 2011;137:687-694.

41. Igawa S, Kasajima M, Ishihara M, et al. Comparison of the efficacy of gefitinib in patients with non-small cell lung cancer according to the type of epidermal growth factor receptor mutation. Oncology 2014;87:215-223.

42. Douillard JY, Shepherd FA, Hirsh V, et al. Molecular predictors of outcome with gefitinib and docetaxel in previously treated non-small-cell lung cancer: data from the randomized phase III INTEREST trial. Journal of clinical oncology : official journal of the American Society of Clinical Oncology 2010;28:744-752.

43. Eichler AF, Kahle KT, Wang DL, et al. EGFR mutation status and survival after diagnosis of brain metastasis in nonsmall cell lung cancer. Neuro-oncology 2010;12:1193-1199.

44. Hsiao SH, Lin HC, Chou YT, et al. Impact of epidermal growth factor receptor mutations on intracranial treatment response and survival after brain metastases in lung adenocarcinoma patients. Lung cancer 2013;81:455-461.

45. De Luca A, Carotenuto A, Rachiglio A, et al. The role of the EGFR signaling in tumor microenvironment. Journal of cellular physiology 2008;214:559-567.

46. Pluquet E, Cadranel J, Legendre A, et al. Osteoblastic reaction in non-small cell lung carcinoma and its association to epidermal growth factor receptor tyrosine kinase inhibitors response and prolonged survival. Journal of thoracic oncology : official publication of the International Association for the Study of Lung Cancer 2010;5:491-496. 